\title{
Metabolic diseases and tendinopathies: the missing link
}

\author{
Francesco Oliva1 \\ Silvia Misiti² \\ Nicola Maffulli ${ }^{3}$
}

1 Department of Orthopaedic and Traumatology, University of Rome "Tor Vergata", School of Medicine, Rome, Italy

2 Fondazione IBSA for scientific research, Pambio, Switzerland

3 Department of Musculoskeletal Disorders, School of Medicine and Surgery, University of Salerno, Salerno, Italy, and Queen Mary University of London, Barts and the London School of Medicine and Dentistry, Centre for Sports and Exercise Medicine, Mile End Hospital, London, UK

This third issue of 2014 of Muscles Ligaments Tendons $\mathrm{J}^{1,2}$ is the fruit of the collaboration between IBSA Foundation (www.fondazioneibsa.org) and ISMULT (www.ismult.com) ${ }^{3}$, and it is based on the Forum "Metabolic diseases and tendinopathies: the missing link", held on 21 June 2014 at the Università della Svizzera Italiana in Lugano, Switzerland.

The forum brought together some 200 members of the international scientific community, including physician and surgeons, basic scientists, physiotherapist, sports scientists and a pre- and doctoral students to discuss this topic: a real translational medicine meeting.

We thank everybody involved in the organization of the forum, the attendees and the speakers coming from everywhere. The speakers were invited to contribute to this issue to leave an indelible mark on this topic in the literature.

The Speakers and Chairmen were:

Michele Abate (University G. d'Annunzio, Chieti, Italy) Isabel Andia (Cruces University Hospital, Barakaldo, Spain)

Anna C. Berardi (Spirito Santo Hospital, Pescara, Italy)

Christian Candrian (Canton Hospital System, Lugano, Switzerland)

Andrew J. Carr (Oxford University, Oxford, UK)

Anna Maria Colao (University of Naples, Italy)

Michael Kjaer (University of Copenhagen, Denmark)

Antonio Frizziero (University of Padua, Italy)

Nicola Maffulli (University of Salerno, Italy and Queen Mary University, London, UK)

Francesco Oliva (Tor Vergata University Rome, Italy) Jess G. Snedeker (University of Zurich, Switzerland)

Louis J. Soslowsky (University of Pennsylvania, USA) Michael Hirschmann (University of Basel, Switzerland)
Tendon conditions adversely impact the quality of life of millions of people, yet their causes and healing mechanisms are still unknown. Despite the array of hypotheses made, there is still a large number of factors affecting tendon health that remains unknown. Recently, genetic factors have been widely discussed. Most certainly inherited genes or gene variants make some individuals more susceptible to these conditions, but the increasing prevalence of metabolic disorders in the past few years has driven scientists to investigate the possible connection between metabolic problems and musculoskeletal diseases.

Humans and animals develop and grow under the physiological control of hormones; many soft tissues and bone diseases are associated to hormones diseases during the organisms development and this is clearly known by many centuries. Despite this postulate, soft tissue diseases, as tendinopathies, have been poorly investigated from this point of view.

At present, the scientific compromise to simplify the pathology of tendinopathies is the "Failed Healing Response" paradigm to explain that something malfunctions in the homeostasis of tendons.

But what really happens in the asymptomatic patients before they develop a symptomatic tendinopathy? This still remains an unanswered question ${ }^{4}$.

Our clinical feeling after managing hundreds of patients for upper and lower limbs tendinopathies, and the recent emerging researches, push us to think that, within the multifactorial etiopathogenesis of tendinopathies, associated hormone disorders, even subclinical, deserve a central role.

The metabolic syndrome affects a range of tissues, and may in part be molecularly described as a dysregulation of cytokines, adipokines, and hormones. Consequently, metabolic imbalance may both directly and indirectly influence even tendon structure ${ }^{5-7}$.

This issue is likely the first orchestrated scientific consensus to focus on this aetiopathogenetic hypothesis. We advocate that clinical and basic scientists involved in the study of tendons and tendinopathy explore this novel hypothesis. This is but the beginning.

\section{References}

1. Maffulli N, Oliva F, Frizziero A. Why M.L.T.J.? Muscles Ligaments Tendons J. 2011;1(1):1-2.

2. Maffulli N, Oliva F, Frizziero A. The baby is growing: PubMed Listing for Muscles Ligaments and Tendons Journal. Muscles Ligaments Tendons J. 2013;3(2):58-59.

3. Maffulli N, Oliva F, Frizziero A. Why I.S.Mu.L.T? Muscles Ligaments Tendons J. 2012;1(3):67. 
4. Modesti A, Oliva F. All is around ECM of tendons!? Muscles Ligaments Tendons J. 2013;3(1):1.

5. Abate M, Schiavone C, Salini V, Andia I. Occurrence of tendon pathologies in metabolic disorders. Rheumatology (Oxford). 2013; 52(4):599-608.

6. Oliva F, Berardi AC, Misiti S, Maffulli N. Thyroid hormones and tendon: current views and future perspectives. Concise review. Muscles Ligaments Tendons J. 2013;11;3(3):201-203.

7. Padulo J, Oliva F, Frizziero A, Maffulli N. Muscles, Ligaments and Tendons Journal. Basic principles and recommendations in clinical and field science research. MLTJ. 2013;4: 250-252. 